\title{
FRANCE : ENTRE DISTANCE ET DÉRISION
}

\author{
Guy Lochard ${ }^{1}$
}

On ne peut comprendre les formes successives de la mise en scène de la parole politique à la télévision française que si l'on a présent à l'esprit le poids durablement exercé dans ce pays par l'Etat sur ce média audiovisuel. Découlent de cette mainmise étatique et gouvernementale des moments très tendus ${ }^{2}$, des pratiques surprenantes ${ }^{3}$ et jusque dans une période proche, des dispositifs communicationnels assez contrastés par rapport à la plupart des pays aux régimes analogues. Singulière à cet égard, cette histoire hexagonale de la parole politique télévisuelle est d'autant plus déconcertante que sa mise en scène a été récemment sujette à de brusques réorientations. Aussi, pour prendre vraiment la mesure de la situation contemporaine, est-il nécessaire d'adopter un large regard rétrospectif, cette recontextualisation historique devant être étendue à l'examen d'autres programmes a priori étrangers à la chose politique.

1 Guy Lochard est professeur à l'Université Paris 3-Sorbonne nouvelle.

2 Après Mai 1968, ce sont des dizaines de journalistes qui ont été licenciés ou écartés de l'antenne.

3 Notamment le contrôle direct de l'information par un ministère spécialisé. Voir à ce propos : Jérôme Bourdon, Haute fidélité, Pouvoir et télévision, 1935-1994, Paris, Seuil, 1994.

Recherches en communication, $\mathrm{n}^{\circ} 24$ (2005). 


\section{Un imaginaire de service public}

Contrairement à ce qui est parfois avancé, la logique durable de contrôle qui a pesé en France sur l'information et l'expression politiques à la télévision ne s'explique pas seulement par le tempérament autoritaire de dirigeants interventionnistes, le général de Gaulle en premier lieu. Il s'origine dans le statut rigoureusement et exclusivement public alloué, lors de sa mise en place à la Libération, à ce nouveau média. Un des premiers effets de ce statut adopté dans d'autres pays européens a été le développement d'un imaginaire particulièrement prégnant de service public ${ }^{1}$ dont la durable vivacité s'enracine dans une histoire ancienne où se mêlent, ainsi que le note Pierre Musso, divers apports « de Colbert à mai 68 en passant par la Révolution française, le Front populaire et la Libération $»^{2}$.

Confondue avec le principe du monopole public, cette conception a pu d'autant plus s'installer que, prise dans le mouvement des grands projets industriels initiés dès l'après-guerre par l'Etat, la télévision a été dans ce pays au centre d'une bataille économique dont l'enjeu était la définition d'un standard international de diffusion. Le retour au pouvoir en 1958 du général de Gaulle ne fera ainsi que renforcer cette emprise étatique sur le nouveau média. Le nouveau président s'implique dans l'adoption de la norme Secam pour la télévision en couleurs. Il réaffirme simultanément le monopole de l'Etat dans l'audiovisuel. Et il renforce dans le même mouvement un strict contrôle gouvernemental sur l'information télévisée poursuivant une instrumentalisation présente dès la $\mathrm{IV}^{\circ}$ République.

Une première leçon se dégage donc de ce retour aux temps pionniers du média. Du fait de l'histoire politique mouvementée qu'elle a traversée au milieu des années 50, la France a été le théâtre d'une confusion entre le rôle de l'Etat en tant que gestionnaire et législa-

1 Une autre conséquence de l'ancrage fort du service public dans l'imaginaire national est le fait que le média télévisuel ait pu être pensé comme devant être mis au service d'un projet éducatif et culturel. Cette aspiration a été déstabilisée dans les années 80 par le processus de privatisation. La télévision reste toutefois encore aujourd'hui largement définie en France comme un possible vecteur de partage de la culture nationale et d'éducation citoyenne. En témoigne encore très récemment (décembre 2002) la proposition dans le Rapport Clément d'une inscription du service public dans le préambule de la Constitution française.

2 Pierre Musso, «Sur la définition du service public », Dossiers de l'audiovisuel 39, Paris, INA-La Documentation française, 1994, p. 21. 
teur d'un nouveau média de communication et un rôle auto-attribué de tuteur de l'information politique. Cette logique de contrôle a atteint son paroxysme avec le retour au pouvoir du général de Gaulle qui s'est approprié ce media pour en faire, contre une presse écrite perçue comme hostile, un instrument direct et étroitement contrôlé de pouvoir. Il faut retenir toutefois qu'elle a présidé plus généralement aux conceptions de tous les dirigeants français, et ce de façon durable ${ }^{1}$.

\section{Une relative démocratisation}

Ce cadre particulièrement rigide de contraintes politiques et administratives explique, qu'à la différence de nombreux pays démocratiques, c'est jusqu'au début des années 70 , qu'a prévalu à la télévision française, un système monologal pour l'expression politique. Un premier basculement s'opère dans la période post-gaulliste où se fait jour timidement un régime dialogal ${ }^{2}$ qui se traduit par l'expérimentation de nouvelles formes de débats puisant dans divers archétypes pour se doter d'une plus grande force d'attractivité. Ainsi L'avocat du diable (1970) dont le dispositif s'inspirait des pratiques judiciaires de l'Eglise. La brièveté de ce programme (six éditions) pointe les limites de cette politique d'ouverture pluraliste, s'incarnant également dans la formule du « duel » qui s'épanouit dans la période suivante avec À armes égales (1970-1973) puis Les 3 vérités (1973-1974).

Si l'existence de ces deux émissions mensuelles atteste une relative mais incontestable démocratisation du débat public à la télévision française, il inaugure tout autant, ainsi que le souligne Noël $\mathrm{Nel}^{3}$, un premier processus de «personnalisation du politique ». De

1 Outre les pratiques interventionnistes encore observables aujourd'hui, on évoquera la tentative tardive de Parlons France, initiée en 1984-85 par Laurent Fabius (alors Premier ministre de François Mitterrand) qui se faisait interroger sur ses projets par un journaliste coopératif.

2 Avec le timide mouvement de l'information amorcé sous la responsabilité de Pierre Desgraupes qui se voit confier en 1969 la mise en place d'une unité autonome d'information. Mais la mainmise du pouvoir en place sur le secteur de l'information est encore réaffirmée en 1972 par Georges Pompidou rappelant que la Voix de l'ORTF est la voix de la France

3 «À armes égales affiche la volonté de transformer l'homme politique d'objet en véritable sujet, de ne plus le considérer comme objet connu, symbole de ce qu'il représente, porte-parole de son parti ou de son ministère. Il va s'agir de favoriser l'expression sans contrainte selon sa personnalité propre». N. Nel, À fleurets mouchetés, 25 ans de débats télévisés, Paris, INA-La documentation française, 
tels programmes permettent en effet l'expression de dirigeants qui campent progressivement leurs personnages dans l'espace médiatique, au même titre d'ailleurs que certaines autorités intellectuelles, syndicales, morales et religieuses également invitées. Tout aussi remarquable que ce processus est, dès cette période, la recherche d'une intégration, dans ces programmes, de la parole de représentants de la collectivité nationale. Esquissée dès le début des années 60 avec Faire face (196062), cette logique d'élargissement est manifeste dans À armes égales, dont le duel est amorcé par la présentation du résultat d'un sondage et relancé par des questions de divers regroupements d'individus donnés comme représentatifs de la population française. Elle est encore plus sensible encore dans Hexagone (1970-1972) qui se présente comme un magazine de société mais qui conserve une place déterminante à la parole de responsables politiques. Fondé sur la formule du dossier renseigné par des reportages de terrain, ce programme permet à des anonymes, sélectionnés pour leur « représentativité », d'interpeller sur les problèmes qu'ils rencontrent dans leur quotidien (le logement, la retraite, la santé) des autorités (membres du gouvernement, commissaire ou délégués de l'Etat, élus) souvent relayés par des experts ${ }^{1}$. De telles émissions demeurent néanmoins, et en dépit de cette recherche d'effets «d'authenticité » et de démocratie, dominées par la parole d'hommes politiques qui, selon le modèle partisan traditionnel, s'expriment en tant que représentants de leurs « camps » respectifs.

\section{Psychologisation et éclosion des forums}

Tel n'est plus le cas dans la période suivante qui est le cadre de changements radicaux dans les domaines conjoints du politique et du télévisuel. Le changement de majorité en 1981 se traduit par une loi qui consacre la fin du monopole d'Etat sur la diffusion et favorise, avec la multiplication des canaux, la diversification de l'offre de programmes. La concurrence qu'introduit ce processus entre les chaînes anciennes et nouvelles, publiques et privées, favorise l'éclosion de dispositifs

1988, p. 35

1 «Ainsi Hexagone se présente donc comme la chambre d'écho et d'analyse de tous les grands problèmes sociaux du moment. À chaque émission, il s'agit fondamentalement de traiter un thème dans ses aspects majeurs, de faire entendre à ce sujet tous les points de vue et de les illustrer par des reportages de qualité ». N. Nel, ibidem, p. 43. 
accentuant la psychologisation de la communication politique. En intervenant comme des rituels à fonction initiatrice ou confirmatrice pour les personnalités politiques conduites de plus en plus à développer un "travail de stylisation » de leurs personnages ${ }^{1}$, L'heure de vérité (A2, 1982-1995) et Questions à domicile (TF1, 1985-1991), représentent à ce titre deux orientations alternatives ${ }^{2}$ de ce nouveau mode de présence de la parole politique à la télévision française. Produits par deux chaînes en concurrence frontale, ces deux programmes illustrent par ailleurs une même dramaturgie triangulaire de la communication politique dans laquelle les journalistes se construisent comme les indispensables médiateurs entre les gouvernants et l'opinion publique dont ils se présentent à la fois comme les seuls exégètes légitimes et les incontournables porte-parole.

C'est le marquage persistant de cet écart entre une parole citoyenne simplement consultée et celle, emphatisée d'élites politiques et médiatiques qui semble de plus en plus faire problème au début des années 90. Différents éléments viennent se conjuguer pour alimenter une mise en question de ce modèle asymétrique ${ }^{3}$ jusque-là faiblement discuté en France. L'un d'eux tient aux transformations qu'ont connues précédemment les magazines dénommés classiquement « de société ». La période antérieure (1985-90) avait été marquée, avec l'éclosion des talk-shows ${ }^{4}$,

1 Erik Neveu donne quelques exemples marquants de ce processus : « Les variantes sont nombreuses de l'invité cultivant un attribut valorisé (Pasqua et la méditerranéité roublarde) aux tactiques de ravalement d'image (Joxe en homme affable et détendu) en passant par le souci d'une entrée réussie (Juppé substituant sa "timidité" et sa "panique" de devenir un professionnel de la politique à une image d'apparatchik) ». Erik Neveu, «L'heure de vérité ou le triangle de la représentation ». Mots, $\mathrm{n}^{\circ} 20, \mathrm{La}$ politique à la télévision, Presses de la Fondation Nationale de sciences politiques, Septembre 1989, p. 70.

2 Comme le remarquent très justement Brigitte Le Grignou et Erik Neveu « L'heure de vérité a su condenser les séductions de la grand-messe médiatique et les mises en scène télématiques d'un lien interactif avec son public... La stratégie alternative ne pouvait jouer qu'à contre-pied. L'intimisme contre la solennité, une dimension plus personnelle face aux questions convenues du débat traditionnel. Le coup de génie d'Alexandre Tarta aura été de traduire cette obligation dans une forme originale : le domicile de l'homme politique ». Brigitte Le Grignou, Erik Neveu, «Emettre la réception ", Réseaux, $\mathrm{n}^{\circ}$ spécial, Sociologie de la télévision en Europe, 1991.

3 Analysé notamment par Jean Mouchon, La politique sous l'influence des médias, Paris, L'Harmattan, Collection "Communication”, 1998.

4 Voir à ce propos Guy Lochard et Jean-Claude Soulages, «Les imaginaires de la parole télévisuelle, Mutations et conflits », Sociologie de la communication (sous la dir. de P. Beaud, P. Flichy, D. Pasquier et L. Quéré), CNET-Réseaux, 1997 
par la promotion d'un nouveau mode de gestion de la parole couplée à l'éclosion d'une série de thématiques bannies jusque-là des écrans car réputées trop conflictuelles ou triviales. Dévaluant la forme cérémonielle et la tournure abstraite du débat d'experts ${ }^{1}$, cette émergence prépare le succès ultérieur d'une première génération de reality shows qui traversent la programmation française au début des années 90. Centrées sur des récits de vie privée, ces émissions poussent à bout une logique de dévoilement d'expériences personnelles en flattant, selon leurs détracteurs, le voyeurisme des téléspectateurs. Elles ont recours à diverses formes d'exhibition de la vie intime des individus (aveu public de fautes, médiation de conflits à l'intérieur des couples²) tout en procédant à une glorification des conduites d'individus anonymes brusquement héroïsés ${ }^{3}$.

En dépit de leur succès populaire, ces émissions vont susciter, tout au long de leur diffusion, un intense débat. Il préfigure celui survenu en 2001-2002 à propos de Loft Story ${ }^{4}$ mais il est surtout très symptomatique de la mutation stratégique que connaît alors la télévision française affichant de plus en plus ses prétentions à l'écoute et à la prise en charge des souffrances individuelles et collectives. De telles logiques de programmation ne sont ainsi pas étrangères à la deuxième crise que connaissent en France les magazines politiques au milieu des années 90. Un signe annonciateur en est, en 1991, le lancement par la Cinq (propriété alors de Berlusconi) de l'émission Les absents ont toujours tort dont tout le dispositif s'inscrit en faux contre les émissions politiques traditionnelles en optant pour une représentation -directe et non plus médiatisée par les instances journalistiques et leurs adjuvants (sondages, SVP) - de l'opinion publiques.

1 Emblématisé en France par Les dossiers de l'écran, émission historique de la télévision française (1967-1991).

2 Un titre significatif : L'amour en danger.

3 Exemple-type : Les marches de la gloire.

4 Voir à ce propos le numéro hors série de la revue Médiamorphoses, « La télé-réalité : un débat mondial » ( sous la direction de Guy Lochard et Guillaume Soulez), Paris, INA-PUF, 2003.

5 Dans une analyse très argumentée par son relevé d'indices, Eric Darras a bien mis en évidence l'importance de cette rupture au plan symbolique qui se traduit essentiellement par une confusion dans le même espace des profanes et des professionnels de la politique. Mais il a aussi montré les limites de ce «simulacre de démocratie directe ». Eric Darras, «Un paysan à la télé, Nouvelles mises en scène du politique », Réseaux, n ${ }^{\circ} 63$, Paris, CNET, 1994, p.77-100. 
Il n'est pas indifférent de constater enfin que, la même année, FR3 lance, dans un créneau horaire moins exposé, Français, si vous parliez dont le titre traduit, explicitement, ce projet de « restitution au peuple français » d'une parole dont la monopolisation par les professionnels de la politique est de plus en plus dénoncée par les commentateurs. Il en va de même de Médiations (TF1, 1986-1992) qui, diffusée plus précocement, procède d'une même volonté affichée de mise en présence directe du profane et du personnel politique. La rupture symbolique qu'instaurent de tels formats est enfin confirmée par un programme exceptionnel organisé de façon précipitée par Antenne $2^{1}$ lors des manifestations étudiantes de mars 1994 contre le CIP². Placé sous la responsabilité de Michel Field, le débat dont il est le cadre confronte directement le ministre des entreprises, Alain Madelin, à une assemblée surchauffée de jeunes l'interpellant directement. Comme l'attestent les chiffres d'audience et les nombreux échos de presse, cette émission fait événement. Par le spectacle qu'elle offre, tout d'abord, qui s'apparente comme le souligne Le Parisien à « une AG en direct $»^{3}$; mais aussi par l'autocritique publique et en direct qu'elle suscite chez le ministre ${ }^{4}$.

La rentrée 1996 constitue en France le moment de cristallisation de ces logiques croissantes de désinstitutionnalisation de l'espace public télévisuel. Significative de ce mouvement est à cette époque la publication par Le Monde ${ }^{5}$ d'un dossier spécial. Il est consacré « au succès des forums cathodiques » qui vont proliférer dans les trois années suivantes avec des émissions aussi diverses que Du fer dans les épinards (France 2, 1997-98), puis Place de la République ${ }^{6}$ (France 2, 1998-1999) et Prise directe (France 3, 1999-2001) aux titres encore plus évocateurs.

1 Qui a déprogrammé dans l'urgence un film pour concurrencer TF1, lequel, de son côté, décida d'allonger son journal télévisé jusqu'à 21 heures.

2 Contrat d'insertion professionnelle.

3 Le Parisien, 30 mars 1994.

4 Exposé à un feu roulant de questions et de violentes critiques, celui-ci en vint en vint à déclarer qu'il était «d'accord avec l'adage : «à travail égal, salaire égal » concédant que «la société est aujourd'hui bloquée » jusqu'à reconnaître en fin d'émission : « à regarder votre émission, on n'aurait pas dû engager le CIP ».

5 Le Monde Radio-télévision, 1-2 décembre 1996. Particulièrement significatif est l'énoncé ouvrant ce long dossier : «Les émissions politiques s'essoufflent. Les Français sont las des face-à-face entre les hommes politiques et journalistes, tous de la même caste. Les téléspectateurs préfèrent interpeller directement les décideurs. Paul Amar, Claude Sérillon et Michel Field leur donnent la parole ».

6 Qui se propose d'être « la caisse de résonance du nouvel état d'esprit de notre société ». Bulletin de presse France 2, 20/10/1998. 


\section{Une radicale redistribution}

Dominant en France à la fin des années 90, le modèle du forum va cependant se vider de sa substance. Dans une conjoncture politique où s'estompe le souvenir du mouvement social de décembre 1995, il se découple des enjeux socio-politiques au profit de thématiques pensées par les directions de programmes comme plus impliquantes et plus fédératrices pour le public, du moins dans les chaînes généralistes. Cette situation s'explique principalement par la définition de nouvelles stratégies de programmation conçues cette fois, non plus au niveau des chaînes, mais à l'échelle des grands groupes d'opérateurs se partageant le marché. Dans un système marqué par la multiplication et la diversification des canaux s'opère en effet, dans chacun de ces ensembles, une redistribution des fonctions qui a pour effet le déplacement des programmes politiques des chaînes principales vers des chaînes d'appoint spécialisées dans l'information.

Le débat politique et sociétal n'a pas, en effet, à cette époque, littéralement disparu des programmes du premier groupe de télévision privé. Il a été déplacé de $T F 1$ vers la chaîne d'information en continu $L C I$ qui programme depuis et régulièrement de nombreux débats sur l'actualité politique nationale, internationale, ainsi que sur la vie intellectuelle. Le raisonnement vaut de même pour le groupe public France Télévisions où c'est pour l'essentiel sur France 5 que les programmes politiques et sociaux ont été délocalisés. Conclure à la disparition de ce type d'émission à la télévision française est donc contraire à la réalité observable. Mais, depuis les années 2000, on doit bien constater une mutation importante dans les modes d'exposition de l'expression politique et sociale, les stratégies de programmation tendant, au nom d'impératifs d'audience, à reléguer cette parole publique dans des chaînes de plus en plus spécialisées, celles évoquées plus haute et plus encore et plus récemment, les chaînes parlementaires Public Sénat et LCP.

Considérant l'audience de ces canaux, cette redistribution relativise donc fortement l'enjeu de ces magazines à base d'échanges dont certains visaient antérieurement, par leurs horaires de programmation et leurs dispositifs, à intégrer dans le débat public un large ensemble de téléspectateurs construits comme des citoyens concernés par les affaires de la Cité. Ce nouveau paysage de programmes confirme en fait et tout autant une érosion amorcée dès la fin des années 80 du pouvoir journalistique à l'intérieur des institutions télévisuelles généralistes. Placés sous contrôle dans les premières décennies de la télévi- 
sion par le pouvoir politique, les journalistes français ont acquis, on a pu le constater, une certaine autonomie au début des années 80 . Cette corporation a dû depuis, tant dans les secteurs public que privé, abdiquer beaucoup de ses ambitions, confrontée qu'elle est à des directions de chaînes donnant la primauté à des animateurs-producteurs qui ont fini par imposer leurs normes dans l'ensemble de la production télévisuelle.

\section{Un comique de situation}

Une traduction du nouveau rapport de force entre ces deux groupes professionnels est la présence banalisée de personnalités politiques dans de nouveaux magazines de divertissement. Rare ou inexistante dans d'autres pays, elle s'est progressivement imposée en France, peu de représentants du monde politique en panne ou en manque d'exposition télévisuelle s'y refusant, en dépit des risques inhérents à ce type de situation. Par delà certaines nuances, le dénominateur commun à toutes ces émissions est en effet la mise en dérision à laquelle se voient soumises la fonction et la parole de ces élus. Différents procédés y concourent. La recherche délibérée d'une forme de comique de situation qui passe par la provocation de rencontres avec d'autres participants relevant d'univers éloignés du politique (humoristes, comédiens, acteurs de films pornographiques). La fonction perturbatrice dévolue à des animateurs secondaires venant, par leurs interventions incidentes, rompre le cadrage sérieux de l'échange assuré par l'animateur principal. Ou encore le jeu de familiarité feinte entre l'animateur et son invité (Karl Zéro dans Le vrai journal de Canal +).

De ce type d'exposition et de gestion de la parole politique Tout le monde en parle (France 2) constitue le parangon, décliné également par plusieurs émissions du week-end (On ne peut pas plaire à tout le monde, Vivement dimanche, Union Libre, etc.). Dans tous ces programmes des chaînes de service public, ce ne sont plus les publicisations d'expériences, et encore moins le fil d'une argumentation politique qui sont au centre des échanges. C'est essentiellement la performance discursive des protagonistes, plongés dans un jeu de rôles dont l'enjeu est le maintien et la valorisation de leur face, confrontés qu'ils sont à diverses formes de stabilisation ou de provocation. Même s'il peut être maîtrisé, le risque de se produire dans ce type d'émission n'apparait donc pas nul pour les personnalités politiques car, plus que les autres types de participants, elles s'y voient avant tout soumises à une forme délibérée 
de déstabilisation induite par la mise en contiguïté avec d'autres participants aux identités excentrées par rapport à la sphère politique.

Ainsi, dans Tout le monde en parle (France 2, 10/06/2000), Alain Madelin succédant sur le plateau à une mannequin, Noémie, qui vient d'être soumise à une interview "poussée » où elle a été interrogée sur ces préférences en matière sexuelle.

Thierry Ardisson : On accueille maintenant Alain Madelin. Alain Madelin Bonsoir, on s'est pas foutu de vous ce soir, j'veux dire là.

Baffie : ce que tu détestes dans l'amour?.

Cette stratégie de mise en dérision des politiques apparaît d'autant plus concertée qu'elle vient, comme on peut le voir dans l'échange mentionné, se coupler avec un procédé de triangulation de l'échange conversationnel, du fait de la fonction perturbatrice dévolue à un animateur secondaire (Baffie dans le programme évoqué) venant, par ses interventions latérales, sans cesse rompre le cadrage sérieux de l'interview assurée par l'animateur principal. En témoigne l'échange suivant dans la même émission :

Alain Madelin : Si un jour on fait un débat sérieux dans lequel on peut s'expliquer, avis à tout le monde, je suis volontaire pour un tel débat...

Baffie : Allez, roule un pet.

Alain Madelin : Je trou...ve...

Baffie : Thierry, donne les cartes, allez on va se détendre, allez, allez ! ! !

Alain Madelin : Sérieusement ! sérieusement, la loi de 70, elle est inappliquée, elle est inapplicable. Je pense pour ma part qu'il faut avoir le courage de cesser d'avoir cette politique de l'autruche qui consiste à ne pas regarder les choses en face. Et que les hommes politiques devraient avoir le courage d'ouvrir le dossier de cette loi de 1970, voilà !

Baffie : Obsédé par les autruches, hein !?

Autre exemple : le traitement administré à l' ancien ministre Claude Allègre (édition du 14/10/2000) :

Thierry Ardisson : Non mais vous êtes un grand savant !

Claude Allègre : Oui enfin, bon... 


\section{Thierry Ardisson : Si !}

Baffie : Si si, mais enfin si c'est bien, on peut être un mauvais ministre et un grand savant!

Claude Allègre : Voilà, voilà...

Baffie : C'n.

On touche ainsi avec ce type d'échange à la finalité de ce type de dispositif conversationnel qui préside aussi à l'émission concurrente On ne peut pas plaire à tout le monde (France 3) et à cette émission apparentée qu'est Vivement dimanche (France 2). Remarquons en effet, que si la situation interlocutive se complexifie, dans cette dernière, avec la présence d'un trio de comparses (aux registres humoristiques assez divers ${ }^{1}$ ) sollicités par l'animateur principal pour dialoguer avec l'invité, l'enjeu de cet échange multilatéral est bien, là aussi, ce constant balancement entre des énonciations sérieuses et humoristiques présent dans les autres programmes cités. Encore qu'il faille, répétons-le, retenir une différence sensible entre les modes de dérision convoqués (et donc de niveau de menaces sur les faces positive et négative des participants) explicables par la variable de l'heure de programmation et les attentes du public présupposées par les instances de production ${ }^{2}$.

Concurrent pour certaines composantes du public avec les journaux télévisés traditionnels, et relevant a priori d'un autre genre, Le vrai journal (Canal+) vérifie de son côté ce parti-pris d'ambiguisation. Du moins dans la séquence d'interview qui en est un point fort. Car si celle-ci revêt une dimension parodique par son décor solennisé caractéristique des entrevues avec les «grands de ce monde », elle repose fondamentalement sur un jeu de familiarité feinte entre l'animateur et son invité, le point d'ancrage étant constitué par un tutoiement réciproque qui rompt avec la déférence affichée publiquement avec les représentants du pouvoir. Ce programme joue donc pleinement lui aussi sur une stratégie pseudo-provocatrice à base de questions et assertions

1 Celui par exemple de l'humour absurde chez Philipe Geluck, de l'ironie grinçante chez Gérard Miller, démissionné aujourd'hui ou encore celui, plus bonhomme, de Bruno Masure, lui aussi remplacé.

2 Vivement dimanche est programmé en effet à une heure d'écoute très fédérative, (le dimanche après-midi), donc à un moment correspondant aux réunions familiales, aux visites entre amis, aux réunions municipales où les politiques peuvent " se laisser aller" en se montrant sous un autre jour. Tout le monde en parle est programmé le samedi soir à une heure tardive : celle des sorties, du règne de la nuit et de ses débordements. 
auxquelles s'efforce de réagir l'invité en témoignant de sa tolérance à l'égard de cette transgression des normes de l'interview politique.

À preuve l'échange suivant (édition du 27/01/02 ):

Karl Zero : Pas plus que ça ? parce qu'il y a un portrait robot qui a été fait par Alain Juppé ton ami, du futur Premier ministre, il dit jeune, dynamique, compétent, il ne va pas parler de lui, donc c'est forcément toi ?

Nicolas Sarkozy : Je te remercie de cette contribution signalée à la paix dans les familles et à l'entente entre Alain Juppé et moi. Mais il y a un détail que tu n'as pas noté, c'est qu'avant cela, il faut gagner.

Par-delà ces variantes, toutes les émissions évoquées ci-dessus proclament ainsi que l'acquisition ou le maintien de la notoriété et de la popularité par l'exposition télévisuelle reste ouvert aux personnalités politiques. Mais que cette ambition a aujourd'hui un coût : le refus du sérieux et l'aptitude à l'autodérision, d'autant plus chers à payer que celles-ci se réclament de responsabilités et du sérieux de leur fonction. Convaincus de l'intérêt de ces investissements médiatiques, rares sont les protagonistes politiques qui se refusent à passer ces réputées épreuves cathodiques dont le bénéfice escompté est évidemment un plus de visibilité pour certains, le regain de celle-ci pour d'autres. Force est de constater qu'en dépit du concert de déplorations consécutives aux dernières présidentielles ${ }^{1}$, ce type de programmes s'est aujourd'hui durablement installé en France dans les pratiques de communication politique ${ }^{2}$, leur présupposé commun étant qu'ils peuvent contribuer à « humaniser» les personnalités publiques en les montrant sous leur face privée.

Une question se pose cependant. À travers la confusion qu'elles offrent entre des énonciations sérieuses et humoristiques, les émissions que l'on vient d'évoquer n'illustrent-elles pas, plus banalement et plus

1 Témoin l'indignation d'Anne Sinclair à l'égard de Tout le monde en parle dans son journal des présidentielles : «Les responsables de cette émission ont fait beaucoup de mal à la perception de la politique que des millions de téléspectateurs regardent désormais comme des voyeurs invités pour un soir au bordel. C'est moins la faute d'Ardisson que celle de tous ses invités, fascinés par le soufre et se pensant plus fort que les autres ». Anne Sinclair, Caméra subjective, Paris, Grasset. 2002, p. 346.

2 Le traumatisme des dernières présidentielles dépassé, les hommes politiques se sont à nouveau précipités dans ces émissions. 
dangereusement, la double visée constitutive de l'infotaiment : offrir simultanément des garanties quant au spectacle (humoristique en l'occurrence) proposé, et des gages quant à la « vérité » des personnes et des faits placés sous la loupe cathodique ? Exemplaire est à ce titre le dispositif du Vrai Journal (Canal +) qui fait se succéder des reportages d'investigation commentés par les journalistes, des sketches parodiques sur l'actualité politique mettant en scène des personnalités politiques et des interviews en plateau des mêmes individus.

\section{Un évitement du débat public}

En procédant à cet examen chronologique élargi, on a donc perçu, sur une dizaine d'années, deux glissements successifs dans la mise en scène de la parole politique à la télévision française. Le premier concerne la minoration, spectaculaire dans un premier temps puis remise en cause, de la parole des seuls élus, délégués et experts au profit d'une parole anonyme, ce phénomène ayant, du moins durant une période, œuvré incontestablement à un élargissement de l'espace public médiatisé. La seconde de ces mutations concerne la privatisation accentuée de la parole politique couplée à sa mise en dérision dans des dispositifs littéralement démagogiques, partie prenante de stratégies plus générales de programmation de plus en plus tentées par un évitement de la fonction dévolue à la télévision généraliste de mise en représentation du débat public.

Si l'on fait exception de ces nouveaux programmes de divertissement, la télévisualisation de la parole politique et sociale ne subsiste en effet qu'à travers quatre modes d'exposition qui soulèvent autant d'interrogations. Tout d'abord, les interviews en studio de personnalités dans les journaux télévisés ou dans des émissions spécialisées comme les Quatre vérités (France 2) qui perpétuent le dialogue exclusif entre élites journalistiques et personnel politique. En second lieu, les magazines classiques comme 100 minutes pour convaincre (France 2) qui semblent vouloir prolonger le modèle asymétrique classique en instrumentalisant la parole citoyenne dans des reportages illustratifs ou en bornant celle-ci à une pure énonciation questionnante, On s'y attardera un instant car ce dispositif privilégié en France a fait éclater au grand jour ses contradictions le 14 avril 2005 avec la prestation offerte par Jacques Chirac dans une émission exceptionnelle diffusée sur TF1, avant le référendum sur l'Europe. Comme nombre d'observateurs l'avaient noté, ce programme, pourtant commandité et formaté par ses 
propres conseillers ${ }^{1}$, devait, à l'encontre des « débats d'initiés » fustigés par Jacques Chirac lui-même, favoriser un dialogue direct de celui-ci avec son opinion publique. Il a surtout mis en lumière, non plus un décalage mais un véritable gouffre entre les attentes et l'état d'esprit de la jeunesse française ${ }^{2}$ et les dispositions du Président de la République, donnant alors toute sa pertinence à l'expression de « monarque républicain ». Troisième type de format observable : les débats mêlant politiques et experts (Ripostes, $C$ dans l'air, France 5 ; Mots croisés, France 2). À base de parole argumentative, mais généralement trop abstraite, ils reproduisent malgré eux une modèle élitiste en cantonnant la parole citoyenne dans une posture de spectatrice réactive par le biais notamment de la mise en scène toujours plus accentuée d'échanges interactifs (par SMS, courriels et appels téléphoniques) avec le public. Quatrième fenêtre enfin qu'il faut aussi évoquer : les magazines dits confessionnels emblématisés par Ça se discute. Travaillés pour certains dossiers par des problématiques politiques latentes, ils sont centrés sur la parole des anonymes. Mais celle-ci, très encadrée, y reste le plus généralement trop émotionnelle pour autoriser les indispensables distanciations et la nécessaire «montée en généralité » des thématiques proprement politiques qui y sont traitées.

Une telle situation semble ainsi ne donner que plus de relief et de prix à des programmes suggérant de possibles moyens d'articulation entre l'espace des préoccupations immédiates du grand public populaire et l'espace médiatique de délibération et de décision politique. Des émissions disparues aujourd'hui comme Ce qui fait débat ${ }^{3}$ ou actuel-

1 Principalement sa fille Claude. L'émission se déroulait dans la salle des fêtes de l'Elysée et mettait en présence Patrick Poivre d'Arvor en maître de cérémonie, deux animateurs, complémentaires Marc-Olivier Fogiel et Jean-Luc Delarue et 83 jeunes de 18 à 30 ans sélectionnés par la Sofres. Elle a rassemblé en moyenne 7.375.360 téléspectateurs, avec une part de marché de 30,8\%.

2 Le moment clef à cet égard fut celui où il déclara que le «pessimisme » des jeunes gens sur la France, sur l'Europe, lui faisait « de la peine » en réaffirmant « Je ne le comprends pas».

3 France 3, Animateur-Producteur: Michel Field (2000-2001). Elle a été analysée en ce sens par Jocelyne Arquembourg-Moreau s'adossant en l'occurrence à la problématique de la construction des problèmes publics de Joseph Gusfeld. Au terme d'un examen du dispositif de ce programme, elle concluait qu' «En cristallisant ce type de conflit, Ce qui fait débat se démarque des grands débats d'opinion en vigueur sur les chaînes françaises dans les années 60-70. Elle se propose d'être le lieu d'une mise en présence d'acteurs sociaux qui déborde le cadre de la formation d'une opinion (ce qui peut se limiter au «quant à soi ») pour déboucher sur une 
lement à l'écran comme Etats généraux (France 5) attestent bien en effet que ces voies existent et qu'elles peuvent œuvrer à la construction d'un espace public concret et participatif donnant toute sa raison d'être à la télévision en tant que lieu de médiation sociale. Mais elles semblent vouées à n'occuper que des créneaux horaires marginaux dans les chaînes généralistes ou à une diffusion dans les canaux dits « de complément ».

demande d'action ». Jocelyne Arquembourg-Moreau, « Ce qui fait débat», Les débats publics en Europe (sous la dir. de Guy Lochard), Paris, L'Harmattan, 2005. 
